\title{
PU(2) MONOPOLES AND A CONJECTURE OF MARIÑO, MOORE, AND PERADZE
}

\author{
P.M.N. Feehan, P.B. Kronheimer, T.G. Leness, and T.S. Mrowka
}

\section{Introduction}

The purpose of this note is to show that some of the recent results of Mariño, Moore, and Peradze [18], [17] can be understood in a simple and direct way via a mechanism pointed out in [2], [4], [6], using the $\mathrm{PU}(2)$-monopole cobordism of Pidstrigach and Tyurin [20].

Throughout this paper, let $X$ denote an oriented smooth four-manifold with $b_{2}^{+}(X)>1$ and with $b^{1}(X)=0$. Once an orientation of $H_{+}^{2}(X)$ is chosen, we can define the Seiberg-Witten invariants which we view as a function

$$
S W_{X}: \operatorname{Spin}^{c}(X) \rightarrow \mathbb{Z}
$$

where $\operatorname{Spin}^{c}(X)$ denotes the set of isomorphism classes of $\operatorname{spin}^{c}$ structures on $X$. Let $S=S(X) \subset \operatorname{Spin}^{c}(X)$ be the support of $S W_{X}$. A cohomology class $K \in H^{2}(X ; \mathbb{Z})$ is called an $S W$-basic class if $K=c_{1}(\mathfrak{s})$ for some $\mathfrak{s} \in S$. Let $B=B(X) \subset H^{2}(X ; \mathbb{Z})$ be the set of SW-basic classes. Given an integral twodimensional cohomology class $w$, we combine the Seiberg-Witten invariants into an analytic function of $h \in H_{2}(X ; \mathbb{R})$ by the formula

$$
\mathbf{S} \mathbf{W}_{X}^{w}(h)=\sum_{\mathfrak{s} \in S(X)}(-1)^{\frac{1}{2}\left(w^{2}+c_{1}(\mathfrak{s}) \cdot w\right)} S W_{X}(\mathfrak{s}) e^{\left\langle c_{1}(\mathfrak{s}), h\right\rangle} .
$$

Let $B^{\perp} \subset H^{2}(X, \mathbb{Z})$ be the orthogonal complement of the basic classes. We say that a four-manifold is abundant if the intersection form on $B^{\perp}$ contains a hyperbolic sublattice. Define the characteristic number

$$
c(X)=-\frac{1}{4}(7 \chi(X)+11 \sigma(X)) .
$$

If $X$ is a complex surface then $c(X)=\chi_{h}(X)-c_{1}^{2}(X)$, where $\chi_{h}(X)=\chi\left(\mathcal{O}_{X}\right)$. Our main theorem is

Theorem 1.1. Suppose that $X$ is abundant and of $S W$-simple type and that Conjecture 3.1 holds for $X$. Then either $c(X)-3<0$, or for any integral lift $w$ of $w_{2}(X)$, the series $\mathbf{S W}_{X}^{w}$ vanishes to order $c(X)-2$ at $h=0$.

Received January 25, 1999.

PMNF was supported by NSF grant number DMS-9704174 and, through the Institute for Advanced Study, by NSF grant number DMS-9729992; PBK was supported by NSF grant number DMS-9531964; TSM was supported by NSF grant number DMS-9796248 
As explained in $[18, \S 8.1]$, this result constrains the homotopy type ${ }^{1}$ of $X$ in terms of the number $b(X)$ of elements in $B /\{ \pm 1\}$ :

Theorem 1.2. [18, Theorem 8.1.1] Let $X$ be a closed, oriented, smooth fourmanifold with $b_{2}^{+}(X)>1$. If $b(X)>0$ and $X$ obeys the conclusion of Theorem 1.1 , then

$$
b(X)>c(X) / 2 .
$$

We recall that a four-manifold $X$ is said to be of $S W$-simple type if for all $\mathfrak{s} \in S$,

$$
c_{1}(\mathfrak{s})^{2}=2 \chi(X)+3 \sigma(X) .
$$

The examples of Fintushel and Stern [13] imply that the bound in Theorem 1.1 is sharp: for every point along the line $c(X)=$ constant $\geq 2$ in the $\left(c_{1}^{2}, \chi_{h}\right)$ plane there exists a four-manifold $X$ with $\mathbf{S W}_{X}^{w}$ vanishing to order $c(X)-2$. Here, $c_{1}^{2}(X)=2 \chi(X)+3 \sigma(X)$ and $\chi_{h}(X)=\frac{1}{4}(\chi(X)+\sigma(X))$. It is interesting to note that the slope of the line in the $\left(c_{1}^{2}, \chi_{h}\right)$ plane implied by inequality (1.2), namely

$$
c_{1}^{2}(X) \geq \chi_{h}(X)-2 b(X)-1,
$$

does not coincide with the classical Noether or Bogomolov-Miyaoka-Yau lines for complex surfaces.

The vanishing condition in the conclusion of Theorem 1.1 is the statement that $X$ has "superconformal simple type" in the terminology of [18], where it is further conjectured (Conjecture 7.8.1) that all four-manifolds of SW-simple type have this property. Theorem 1.1 therefore reduces the conjecture of [18] for abundant manifolds to the technical Conjecture 3.1. The latter conjecture is the assertion that ideal Seiberg-Witten moduli spaces $M_{\mathfrak{s}}^{s w} \times \operatorname{Sym}^{\ell}(X)$ in any stratum of the compactified moduli space of $\mathrm{PU}(2)$ monopoles $\bar{M}_{W, E}$ make no contribution to the Donaldson invariants defined by $\bar{M}_{E}^{\text {asd }} \subset \bar{M}_{W, E}$ if their associated Seiberg-Witten invariants vanish. This in turn is a simple consequence of a conjecture, attributed to Pigstrigach and Tyurin [2, Conjecture 4.1], that pairings of Donaldson-type cohomology classes with links of ideal Seiberg-Witten moduli spaces in $\bar{M}_{W, E}$ are given by the product of $S W_{X}(\mathfrak{s})$ and a universal polynomial in the intersection form $Q_{X}$ and the classes $c_{1}(\mathfrak{s})-\Lambda$ and $\Lambda$, with coefficients depending only on $\left(c_{1}(\mathfrak{s})-\Lambda\right)^{2},\left(c_{1}(\mathfrak{s})-\Lambda\right) \cdot \Lambda, \Lambda^{2}, S W_{X}(\mathfrak{s})$, and a universal function of $\chi(X)$ and $\sigma(X)$; the polynomial degree depends on $\ell$ and the degree of the Donaldson invariant. (In the paragraph following the statement of this conjecture in $\S 3.1$ we give an informal explanation of why Conjecture 3.1 should hold and in the last paragraphs of $\S 3.1$ and $\S 3.2$, we explain the role of the conjecture in the proof of Theorem 1.1.) The work of the first and third authors $([3],[4],[5],[6],[2],[1],[8],[9],[7])$ goes a long way towards a

\footnotetext{
${ }^{1}$ A similar constraint was conjectured earlier by Fintushel and Stern (private communication; see also [13]).
} 
proof of [2, Conjecture 4.1] and, in particular, Conjecture 3.1. If Conjecture 3.1 is not assumed, a somewhat weaker result with a correction term can still be deduced from those papers; the correction depends on the maximum dimension of the non-empty Seiberg-Witten moduli spaces - see $\S 3$ for more details. The abundance condition is used to construct the right $\mathrm{PU}(2)$-monopole setup and is not the most general condition under which the theorem can be proved; see the discussion after Lemma 2.2 .

If one assumes Witten's [21] conjectured formula (2.2) relating the SeibergWitten invariants for manifolds of simple type and the Donaldson invariants, the conclusion of Theorem 1.1 is equivalent to a vanishing theorem for the Donaldson invariants through certain degrees. A restricted version of Witten's conjecture — see Theorem 2.1 below — is proved in [4], [5], [6]. In fact this theorem implies many different relations between the Donaldson and Seiberg-Witten invariants and will be used here in two ways: to prove the vanishing result for Donaldson invariants, Theorem 1.3 below, and to deduce Theorem 1.1, most cases of which follow from Theorem 1.3 and the restricted version of Witten's conjecture.

Theorem 1.3. Assume Conjecture 3.1 and that $X$ is of $S W$-simple type and abundant. Then for

$$
0 \leq d \leq c(X)-1 \quad \text { and } \quad m \geq 0
$$

we have for all $w \in H^{2}(X, \mathbb{Z})$ with $w=w_{2}(X)(\bmod 2)$,

$$
D_{X}^{w}\left(h^{d-2 m} x^{m}\right)=0 .
$$

(See §2 for conventions concerning the Donaldson invariants $D_{X}^{w}$.)

Acknowledgments. We are grateful to Robert Friedman, Peter Ozsváth, and Zoltán Szabó for helpful comments. The first author would like to thank the Institute for Advanced Study, Princeton, the Institut des Hautes Études Scientifiques, Bures-sur-Yvette, as well as the National Science Foundation, for their generous support during the preparation of this article.

\section{The Donaldson invariants and relations to the Seiberg-Witten invariants}

We quickly review some basic definitions regarding the Donaldson invariants (see [16]). Let

$$
\mathbb{A}(X)=\operatorname{Sym}\left(H_{\text {even }}(X ; \mathbb{R})\right)
$$

be the graded algebra, with $z=\beta_{1} \beta_{2} \cdots \beta_{r}$ having total degree $\operatorname{deg}(z)=$ $\sum_{p}\left(4-i_{p}\right)$, when $\beta_{p} \in H_{i_{p}}(X ; \mathbb{R})$. In particular a point $x \in X$ gives a distinguished generator still called $x$ in $\mathbb{A}(X)$ of degree four. For any choice of $w \in H^{2}(X ; \mathbb{Z})$ there is a corresponding Donaldson invariant which is now a linear function

$$
D_{X}^{w}: \mathbb{A}(X) \rightarrow \mathbb{R}
$$


and is defined by evaluating cohomology classes corresponding to elements of $\mathbb{A}(X)$ on instanton moduli spaces of $\mathrm{SO}(3)$-bundles $P$ with $w_{2}(P) \equiv w(\bmod 2)$, $w$ determining the orientation of the moduli spaces using Donaldson's conventions $\left[16, \S 2(\right.$ ii) $]$. If $w \equiv w^{\prime}(\bmod 2)$ we have

$$
D_{X}^{w}=(-1)^{\frac{1}{4}\left(w-w^{\prime}\right)^{2}} D_{X}^{w^{\prime}}
$$

Also $D_{X}^{w}(z)=0$ unless $z$ contains a monomial $m$ with

$$
\operatorname{deg}(m) \equiv-2 w^{2}-\frac{3}{2}(\chi(X)+\sigma(X))(\bmod 8),
$$

and $\chi(X)+\sigma(X) \equiv 0(\bmod 4)$.

A four-manifold has KM-simple type if for all $z \in \mathbb{A}(X)$ we have

$$
D_{X}^{w}\left(x^{2} z\right)=4 D_{X}^{w}(z) .
$$

It is known that if this relation holds for one $w$, it holds for all $w$. For manifolds of KM-simple type one introduces the formal power series in a variable $h \in H_{2}(X)$,

$$
\mathbf{D}_{X}^{w}(h)=D_{X}^{w}\left(\left(1+\frac{1}{2} x\right) e^{h}\right) .
$$

By Equation (2.1) the series $\mathbf{D}_{X}^{w}$ is an even function if

$$
-w^{2}+\frac{3}{4}(\chi(X)+\sigma(X)) \equiv 0 \quad(\bmod 2),
$$

and is odd otherwise. Notice that $\mathbf{S W}_{X}^{w}$ has the same property since

$$
S W_{X}(\mathfrak{s})=(-1)^{\frac{1}{4}(\chi(X)+\sigma(X))} S W_{X}(\overline{\mathfrak{s}}) .
$$

In addition if $w \equiv w^{\prime}(\bmod 2)$ then

$$
\mathbf{S W}_{X}^{w^{\prime}}=(-1)^{\frac{1}{4}\left(w-w^{\prime}\right)^{2}} \mathbf{S} \mathbf{W}_{X}^{w}
$$

According to [16, Theorem 1.7], when $X$ has KM-simple type the series $\mathbf{D}_{X}^{w}(h)$ is an analytic function of $h$ and there are finitely many characteristic cohomology classes $K_{1}, \ldots, K_{m}$ (the KM-basic classes) and constants $a_{1}, \ldots, a_{m}$ (independent of $w)$ so that

$$
\mathbf{D}_{X}^{w}(h)=e^{\frac{1}{2} h \cdot h} \sum_{i=1}^{r}(-1)^{\frac{1}{2}\left(w^{2}+K_{i} \cdot w\right)} a_{i} e^{\left\langle K_{i}, h\right\rangle} .
$$

Witten's conjecture [21] relating the Donaldson and Seiberg-Witten invariants of manifolds of simple type says that

$$
\mathbf{D}_{X}^{w}(h)=2^{2-c(X)} e^{\frac{1}{2} h \cdot h} \mathbf{S} \mathbf{W}_{X}^{w}(h)
$$

While a complete, mathematically rigorous proof of Witten's conjecture has not yet been obtained, a possible approach using a PU(2)-monopole cobordism was proposed by Pidstrigach and Tyurin [20]. By employing the PU(2)-monopole 
cobordism, the first and third author [4], [5], [6] proved relations between these invariants which we restate here in somewhat restricted form. To state their results define for $\Lambda \in H^{2}(X ; \mathbb{Z})$,

$$
r(\Lambda)=-\Lambda^{2}-\frac{1}{4}(11 \chi(X)+15 \sigma(X)) .
$$

This number keeps track of the depth in the Uhlenbeck compactification of the PU(2)-moduli space that reducibles appear in terms of the degree of the corresponding Donaldson invariant when $X$ has SW-simple type. Also define

$$
i(\Lambda)=\Lambda^{2}-\frac{1}{4}(3 \chi(X)+7 \sigma(X))
$$

This number keeps track of the index of the Dirac operator for the PU(2)-moduli space in terms of the degree of the corresponding Donaldson invariant. If we assume Conjecture 3.1 the results of [4], [5], [6] yield:

Theorem 2.1. [6, Theorem 1.1] Assume that $X$ satisfies Conjecture 3.1 and is of $S W$-simple type. Suppose that $\Lambda \in B^{\perp}$ and that $w \in H^{2}(X ; \mathbb{Z})$ with $w-\Lambda \equiv$ $w_{2}(X)(\bmod 2)$. If we have

$$
\delta<r(\Lambda) \text { and } \delta<i(\Lambda)
$$

then for all $h \in H^{2}(X ; \mathbb{R})$,

$$
D_{X}^{w}\left(h^{\delta-2 m} x^{m}\right)=0
$$

If we instead have

$$
\delta=r(\Lambda) \quad \text { and } \quad \delta<i(\Lambda)
$$

then for all $h \in H^{2}(X ; \mathbb{R})$,

$$
\begin{aligned}
D_{X}^{w}\left(h^{\delta-2 m} x^{m}\right)=2^{1-\frac{1}{2}(c(X)+\delta)}(-1)^{m-1+\frac{1}{2} \Lambda^{2}-\Lambda \cdot w} & \\
& \times \sum_{\mathfrak{s} \in S}(-1)^{\frac{1}{2}\left(w^{2}+c_{1}(\mathfrak{s}) \cdot w\right)} S W_{X}(\mathfrak{s})\left\langle c_{1}(\mathfrak{s})-\Lambda, h\right\rangle^{\delta-2 m} .
\end{aligned}
$$

Since $\Lambda^{2}-2 \Lambda \cdot w=\sigma(X)-w^{2}(\bmod 8)$, the sign factor $(-1)^{\frac{1}{2} \Lambda^{2}-\Lambda \cdot w}$ may also be written as $(-1)^{\frac{1}{2}\left(\sigma(X)-w^{2}\right)}$.

The hypotheses of Theorem 1.1 in [6] do not require that Conjecture 3.1 holds, that $b_{2}^{+}(X)>1$, or that $X$ have Seiberg-Witten simple type. If $b_{2}^{+}(X)=1$ a similar result also holds when the chamber structure is taken into account. See [6, Theorem 1.1] for the most general statements, including a partial treatment of the case when $b_{1}(X)>0$. The role of SW-simple type, the manner in which Conjecture 3.1 strengthens Theorem 1.1 in [6] and the derivation of Theorem 2.1 from it are explained in $\S 3$. The mechanism underlying the vanishing result in Equation (2.5) was pointed out in [4]: see, for example, Theorem 5.33 and the paragraph following Equation (7.2). The fact that the PU(2)-monopole 


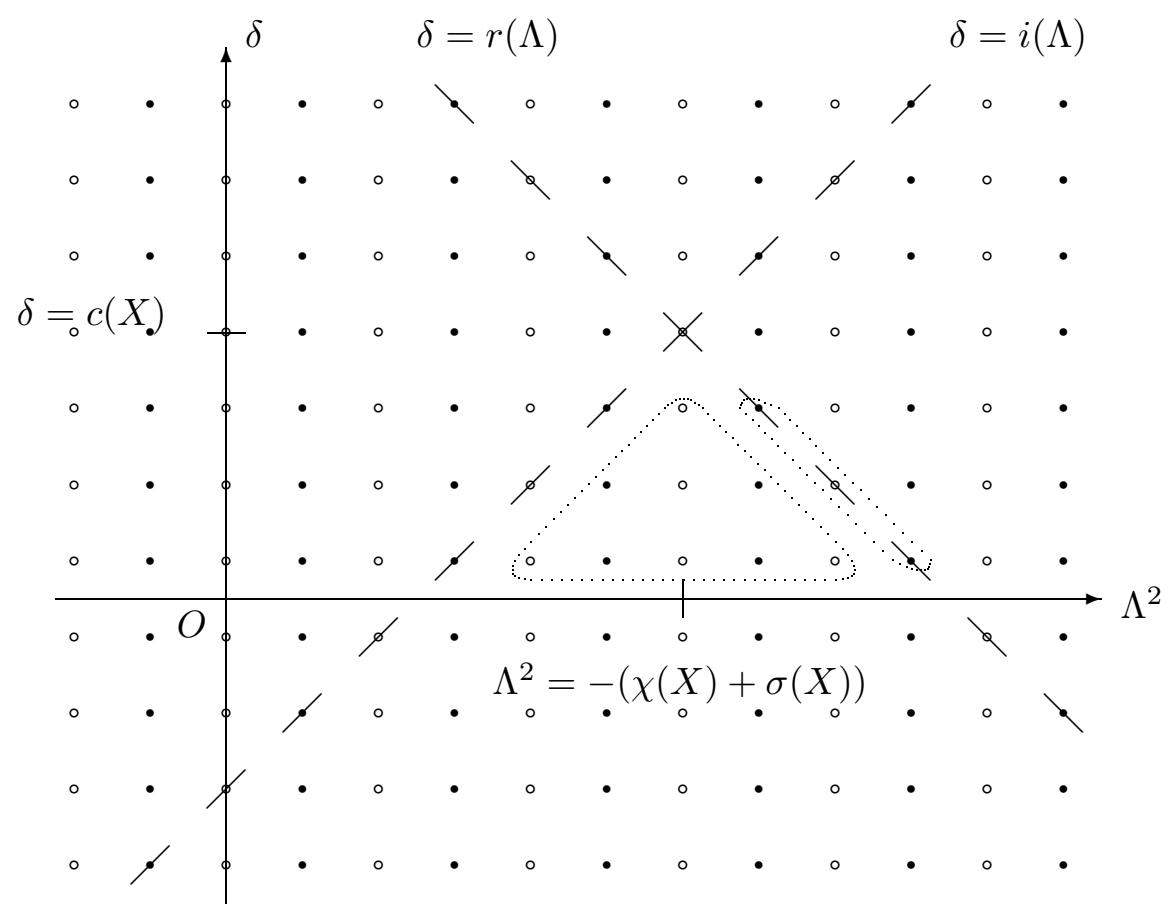

Figure 1

cobordism should imply some relations between the SW-basic classes was evident from Lemmas 5.30 and 5.31 in [4]: see Conjecture 4.1 in [2].

Elements of $B$ are always characteristic, so the condition $\Lambda \in B^{\perp}$ (assuming $B$ is non-empty) means that $\Lambda \cdot w_{2}$ is zero, and $\Lambda^{2}$ is therefore even. Via the additional condition $\Lambda-w \equiv w_{2}(\bmod 2)$, the pair $w$ and $\Lambda$ is constrained so that $w^{2} \equiv \Lambda^{2}+\sigma(X)(\bmod 4)$. Furthermore, if $w$ is characteristic, so $w^{2} \equiv \sigma(X)$ $(\bmod 8)$, and $B$ is non-empty then this condition implies that $\Lambda \equiv 0(\bmod 2)$ and $\Lambda^{2} \equiv 0(\bmod 8)$.

Figure 1 shows a typical picture in the $\left(\Lambda^{2}, \delta\right)$ plane, assuming that $c(X)$ is positive. The lines $\delta=i(\Lambda)$ and $\delta=r(\Lambda)$ intersect at $\delta=c(X)$ and $\Lambda^{2}=$ $-(\chi(X)+\sigma(X))$. The Theorem asserts the vanishing of a Donaldson invariant when $\left(\Lambda^{2}, \delta\right)$ is in the interior of the outlined triangle, and gives a formula for the Donaldson invariant in terms of Seiberg-Witten invariants when $\left(\Lambda^{2}, \delta\right)$ is one of the marked points on the right-hand edge of the triangle.

The marked points are the lattice points with $2 \delta \equiv-2 w^{2}-\frac{3}{2}(\chi(X)+\sigma(X))$ $(\bmod 8)(\operatorname{see}$ Equation $(2.1))$ and $\Lambda^{2} \equiv w^{2}-\sigma(X)(\bmod 4)$. We have drawn the case in which $w^{2}-\sigma(X)$ is zero $\bmod 4$, so that the intersection of the two lines is one of the marked lattice points (because $2 c(X)$ always satisfies the condition (2.1)). In the case that $w$ is characteristic, $\Lambda^{2}$ is constrained to be $0 \bmod 8$ (the white dots); we have drawn the case that $-(\chi(X)+\sigma(X))$ is $0 \bmod 8$ also.

Thus we see that in order to apply Theorem 2.1 we need the restriction of 
the intersection form to $B^{\perp}$ to be rich enough that it realizes particular values, on classes with certain constraints on their mod 2 reductions. The definition of abundant is chosen simply to have some rather general but compact hypothesis under which we can find the required classes. The precise hypothesis needed are the conclusions of the following Lemma.

Lemma 2.2. If $X$ is abundant then there are cohomology classes $\Lambda_{0}, \Lambda_{1} \in B^{\perp}$ with $\Lambda_{0} \equiv \Lambda_{1}(\bmod 2)$ so that:

$$
\Lambda_{0}^{2}=-(\chi(X)+\sigma(X)), \quad \Lambda_{1}^{2}=-(\chi(X)+\sigma(X))+4 .
$$

There is also a class $\Lambda \in 2 B^{\perp}$ with $\Lambda^{2}=-(\chi(X)+\sigma(X))$ if $-(\chi(X)+\sigma(X)) \equiv 0$ $(\bmod 8)$ and $\Lambda^{2}=-(\chi(X)+\sigma(X))+4$ if $-(\chi(X)+\sigma(X)) \equiv 4(\bmod 8)$.

Proof. Since $X$ is abundant we can find $e_{1}, e_{2} \in B^{\perp}$ so that $e_{1} \cdot e_{1}=e_{2} \cdot e_{2}=0$ and $e_{1} \cdot e_{2}=1$. Set $h=\frac{1}{4}(\chi(X)+\sigma(X))$. Then $\Lambda_{0}=2 e_{1}-h e_{2}$ and $\Lambda_{1}=2 e_{1}+(1-h) e_{2}$ will do. If $h \equiv 0(\bmod 2)$ then taking $\Lambda=\Lambda_{0}$ proves the second assertion and if $h \equiv 1(\bmod 2)$ taking $\Lambda=\Lambda_{1}$ proves the second assertion.

There are non-abundant four-manifolds: for example, some of the fake K3surfaces of [14] fail to be abundant. If log transforms are performed on tori in three distinct nuclei then the intersection form on $B^{\perp}$ is a degenerate form with three-dimensional radical and having an $-E_{8} \oplus-E_{8}$ summand. These manifolds however satisfy the conclusion of Lemma 2.2. On the other hand one can show that a simply connected minimal surface of general type is abundant.

Here is the proof of Theorem 1.1 of the introduction.

Proof of Theorem 1.1. Choose $w$ characteristic as in the hypotheses of Theorem 1.1. To prove the theorem we must show that the Taylor coefficients of $\mathbf{S W}_{X}^{w}$ are zero in degrees $d$ with $0 \leq d \leq c(X)-3$. These coefficients are zero unless $d \equiv-w^{2}+\frac{3}{4}(\chi(X)+\sigma(X)) \equiv c(X)(\bmod 2)$ so write $d=c(X)-4-2 m$ for some $m \geq 0$. Take $\Lambda_{0}, \Lambda_{1} \in B^{\perp}$ with

$$
\left.\Lambda_{0}^{2}=-(\chi(X))+\sigma(X)\right), \quad \Lambda_{1}^{2}=-(\chi(X)+\sigma(X))+4
$$

and $\Lambda_{0} \equiv \Lambda_{1}(\bmod 2)$ as provided by Lemma 2.2 . We have

$$
r\left(\Lambda_{0}\right)=c(X)=i\left(\Lambda_{0}\right)
$$

so taking $\delta=d+2 m=c(X)-4$ in Equation (2.5) of Theorem 2.1 (applied to calculate $D_{X}^{w^{\prime}}$, with $w^{\prime}=w+\Lambda_{0}$ and $\Lambda=\Lambda_{0}$ ), one concludes that

$$
D_{X}^{w+\Lambda_{0}}\left(h^{d} x^{m}\right)=0 .
$$

On the other hand we have

$$
r\left(\Lambda_{1}\right)=c(X)-4 \quad \text { and } \quad i\left(\Lambda_{1}\right)=c(X)+4
$$


so taking $\delta=c(X)-4$ again but now using Equation (2.6) (applied to calculate $D_{X}^{w^{\prime}}$, with $w^{\prime}=w+\Lambda_{1}$ and $\left.\Lambda=\Lambda_{1}\right)$, one concludes that

$$
\begin{aligned}
& D_{X}^{w+\Lambda_{1}}\left(h^{d} x^{m}\right)=2^{1-\frac{1}{2}(c(X)+d)-m}(-1)^{m-1+\frac{1}{2} \Lambda_{1}^{2}-\Lambda_{1} \cdot w} \\
& \times \sum_{\mathfrak{s} \in S}(-1)^{\frac{1}{2}\left(w^{2}+c_{1}(\mathfrak{s}) \cdot w\right)} S W_{X}(\mathfrak{s})\left\langle c_{1}(\mathfrak{s})-\Lambda_{1}, h\right\rangle^{d} .
\end{aligned}
$$

Since $\Lambda_{0} \equiv \Lambda_{1}(\bmod 2)$ and $\Lambda_{0}-\Lambda_{1} \in B^{\perp}$ we have $0=D_{X}^{w+\Lambda_{0}}\left(h^{d} x^{m}\right)=$ $D_{X}^{w+\Lambda_{1}}\left(h^{d} x^{m}\right)$. Putting all this together implies that the Taylor coefficients at the origin of the analytic function

$$
e^{-\left\langle\Lambda_{1}, h\right\rangle} \mathbf{S} \mathbf{W}_{X}^{w}(h)
$$

are zero up to degree $c(X)-3$. Since $e^{-\left\langle\Lambda_{1}, h\right\rangle}$ is invertible it must be that the $\mathbf{S W}_{X}^{w}(h)$ vanishes to the required order there.

It remains to prove Theorem 1.3 of the introduction.

Proof of Theorem 1.3. Fix $w$ characteristic. Now there is nothing to prove unless $d \equiv-w^{2}+\frac{3}{4}(\chi(X)+\sigma(X))(\bmod 4)$ so assume this as well. If $-(\chi(X)+\sigma(X)) \equiv$ $0(\bmod 8)$ find $\Lambda \in 2 B^{\perp}$ so that $\Lambda^{2}=-(\chi(X)+\sigma(X))$ as in Lemma 2.2. Then

$$
i(\Lambda)=r(\Lambda)=c(X)
$$

and so for any $d \leq c(X)-4$ we can use Equation (2.5) to conclude

$$
0=D_{X}^{w+\Lambda}\left(h^{d-2 m} x^{m}\right)=D_{X}^{w}\left(h^{d-2 m} x^{m}\right) .
$$

If $-(\chi(X)+\sigma(X)) \equiv 4(\bmod 8)$ find $\Lambda \in 2 B^{\perp}$ so that $\Lambda^{2}=-(\chi(X)+\sigma(X))+4$ as in Lemma 2.2. Then

$$
i(\Lambda)=c(X)+4 \quad \text { and } \quad r(\Lambda)=c(X)-4
$$

and so for any $d \leq c(X)-8$ we can use Equation (2.5) to conclude

$$
0=D_{X}^{w+\Lambda}\left(h^{d-2 m} x^{m}\right)=D_{X}^{w}\left(h^{d-2 m} x^{m}\right) .
$$

While for $d=c(X)-4$ Equation (2.6) applies to give

$$
\begin{aligned}
& D_{X}^{w}\left(h^{d-2 m} x^{m}\right)=2^{1-\frac{1}{2}(c(X)+d)}(-1)^{m+\frac{1}{2} \Lambda^{2}-\Lambda \cdot w} \\
& \times \sum_{\mathfrak{s} \in S}(-1)^{\frac{1}{2}\left(w^{2}+c_{1}(\mathfrak{s}) \cdot w\right)} S W_{X}(\mathfrak{s})\left\langle c_{1}(\mathfrak{s})-\Lambda, h\right\rangle^{d-2 m} .
\end{aligned}
$$

But up to a constant factor the right-hand side of this equation is the Taylor coefficient of degree $d-2 m$ in $h$ of $e^{-\langle\Lambda, h\rangle} \mathbf{S W}_{X}^{w}(h)$ and hence vanishes by the previous theorem.

Notice that it is only the case $-(\chi(X)+\sigma(X)) \equiv 4(\bmod 8)$ and $d=c(X)-4$ which requires the formula (2.6) (and thus [6, Theorem 1.1]); the remaining 
cases are direct consequences of the more elementary vanishing result (2.5) (and thus [6, Theorem 3.33]). Also notice that in many cases the vanishing can be deduced from another mechanism. If there are distinct classes $\Lambda$ and $\Lambda^{\prime}$ so that Equation (2.8) holds then we deduce that $e^{-\langle\Lambda, h\rangle} \mathbf{S} \mathbf{W}_{X}^{w}(h)=e^{-\left\langle\Lambda^{\prime}, h\right\rangle} \mathbf{S} \mathbf{W}_{X}^{w}(h)$ up to order $c(X)-4$ in $h$ and hence $\mathbf{S W}_{X}^{w}(h)$ vanishes to that order.

\section{On Theorem 2.1 and Conjecture 3.1}

We explain how to derive Theorem 2.1 from [6, Theorem 1.1] and Conjecture 3.1 .

3.1. The multiplicity conjecture. The formula in [6, Theorem 1.1] for Donaldson invariants in terms of Seiberg-Witten invariants requires a choice of base $\operatorname{spin}^{c}$ structure $\mathfrak{s}_{0}=\left(W^{+}, W^{-}, \rho\right)$ and Hermitian, rank-two vector bundle $E$ so that reducible $\mathrm{PU}(2)$ monopoles (which we view as Seiberg-Witten monopoles — see [5, §2 \& 3]) appear only in the top level of $\bar{M}_{W, E}$, if at all. However, by assuming Conjecture 3.1, we can significantly relax the preceding constraint and allow reducible PU(2) monopoles to appear in the lower-levels of $\bar{M}_{W, E}$ provided their associated Seiberg-Witten invariants vanish. Hence, the sum in $[6$, Theorem 1.1] over set of $\operatorname{spin}^{c}$ structures defining reducible $\mathrm{PU}(2)$ monopoles lying only in the top level $M_{W, E}$ can be replaced by a sum over $\operatorname{spin}^{c}$ structures whose associated reducibles can lie in the top level of $\bar{M}_{W, E}$ if their Seiberg-Witten invariants are non-zero and in any level if they are zero. This is a much weaker requirement than that of $[6$, Theorem 1.1], which asks that there be no nonempty lower-level Seiberg-Witten moduli spaces, a condition which depends, for example, on the choice of perturbations and is difficult to verify in practice.

By the hypothesis of Theorem 2.1, we are given a class $w \in H^{2}(X ; \mathbb{Z})$ : recall from Equation (2.1) that the Donaldson invariant $D_{X}^{w}(z)$ of $\operatorname{deg}(z)=2 \delta \in 2 \mathbb{Z}$ is defined to be zero unless $\delta \equiv-w^{2}-\frac{3}{4}(\chi+\sigma)(\bmod 4)$. As there is no loss in assuming this, let $p \in \mathbb{Z}$ be determined by the equation

$$
\delta=-p-\frac{3}{4}(\chi+\sigma)
$$

so $p=w^{2}(\bmod 4)$, and define a Hermitian, rank-two vector bundle $E$ over $X$ by requiring that $c_{1}(E)=w$ and $c_{2}(E)-\frac{1}{4} c_{1}(E)^{2}=-\frac{1}{4} p$. For such a bundle $E$, we have $p_{1}(\mathfrak{s u}(E))=p$.

The hypotheses of Theorem 2.1 also provide us with a class $\Lambda \in H^{2}(X ; \mathbb{Z})$. We therefore fix a $\operatorname{spin}^{c}$ structure $\mathfrak{s}_{0}=\left(W^{+}, W^{-}, \rho\right)$ on $X$ with $c_{1}\left(\mathfrak{s}_{0}\right)=c_{1}\left(W^{+}\right)$ determined by

$$
c_{1}\left(W^{+}\right)+c_{1}(E)=\Lambda .
$$

Any other $\operatorname{spin}^{c}$ structure $\mathfrak{s}$ on $X$ defines a class in $H^{2}(X ; \mathbb{Z})$ and a Hermitian line bundle $L_{1}$ over $X$ such that $c_{1}(\mathfrak{s})=c_{1}\left(\mathfrak{s}_{0}\right)+2 c_{1}\left(L_{1}\right)$. We write $\mathfrak{s}=\mathfrak{s}_{0} \otimes L_{1}=$ $\left(W^{+} \otimes L_{1}, W^{-} \otimes L_{1}, \rho\right)$. Hence, the $\operatorname{spin}^{c}$ structure $\mathfrak{s}$ determines a split, rank-two, 
Hermitian vector bundle $E^{\prime}=L_{1} \oplus(\operatorname{det} E) \otimes L_{1}^{*}$ with $p_{1}\left(\mathfrak{s u}\left(E^{\prime}\right)\right)=p_{1}(\mathfrak{s u}(E))$ $(\bmod 4)$ and

$$
p_{1}\left(\mathfrak{s u}\left(E^{\prime}\right)\right)=\left(2 c_{1}\left(L_{1}\right)-c_{1}(E)\right)^{2}=\left(c_{1}(\mathfrak{s})-\Lambda\right)^{2} .
$$

These split bundles, $E^{\prime}$, in turn define families of reducible $\mathrm{PU}(2)$ monopoles.

The vector bundle $E^{\prime}$ defines reducible $\mathrm{PU}(2)$ monopoles in some level of $\bar{M}_{W, E}$ only if $p_{1}\left(\mathfrak{s u}\left(E^{\prime}\right)\right) \geq p_{1}(\mathfrak{s u}(E))$, in which case they would be contained in the level $\ell \in \mathbb{Z}_{\geq 0}$, where

$$
\left.p_{1}\left(\mathfrak{s u}\left(E^{\prime}\right)\right)=p_{1}(\mathfrak{s u}(E))\right)+4 \ell .
$$

Let us first consider the case $\ell>0$, so the corresponding reducible solutions to the $\mathrm{PU}(2)$ monopole equations lie in the level $M_{W, E_{-\ell}} \times \operatorname{Sym}^{\ell}(X)$ of the Uhlenbeck compactification

$$
\bar{M}_{W, E} \subset \bigcup_{\ell=0}^{N} M_{W, E_{-\ell}} \times \operatorname{Sym}^{\ell}(X),
$$

where $E_{-\ell}$ is a Hermitian, rank-two vector bundle over $X$ with $c_{1}\left(E_{\ell}\right)=c_{1}(E)$ and $c_{2}\left(E_{-\ell}\right)=c_{2}(E)-\ell$. The smooth loci $M_{W, E_{-\ell}}^{*, 0} \times \Sigma$ of the lower level $M_{W, E_{-\ell}} \times \operatorname{Sym}^{\ell}(X)$, where $\Sigma \subset \operatorname{Sym}^{\ell}(X)$ is a smooth stratum, have codimension greater than or equal to $2 \ell$. For $z \in \mathbb{A}(X)$, a geometric representative $\bar{V}(z) \subset \bar{M}_{W, E}$ of codimension $\operatorname{deg}(z)$ is defined in [6]. It is shown in [6] that this geometric representative intersects the lower levels of $\bar{M}_{W, E}$ in a set with codimension $\operatorname{deg}(z)$ except at the reducible points. There is also a geometric representative $\bar{W}\left(x^{n_{c_{1}}}\right) \subset \bar{M}_{W, E}$ which has codimension $2 n_{c_{1}}$ on the complement of the reducible and zero-section points. The formula in [6, Theorem 3.33] is proved by computing the intersections of the geometric representatives, $\bar{V}(z) \cap \bar{W}\left(x^{n_{c_{1}}}\right)$, with the links of the strata of the anti-self-dual and reducible $\mathrm{PU}(2)$ monopoles in $M_{W, E}$. Thus to extend [6, Theorem 3.33] to the case where there are reducibles in the lower levels $M_{W, E_{-\ell}} \times \operatorname{Sym}^{\ell}(X), \ell>0$, one needs to consider the links in $\bar{M}_{W, E}$ of the families

$$
M_{\mathfrak{s}}^{s w} \times \operatorname{Sym}^{\ell}(X)
$$

Here, $M_{\mathfrak{s}}^{s w}$ is the perturbation of the standard Seiberg-Witten moduli space described in [5, Equation (2.14)] and [5, Lemma 3.13].

The gluing theorems of [8], [9] provide a sufficiently explicit description of a neighborhood of the family (3.4) that we can define their link in $\bar{M}_{W, E}$, which we denote by $\mathbf{L}_{W, E, L_{1}}$. Given the results of [8], [9], the formula of [6, Theorem 3.33] can then be replaced by

$$
D_{X}^{w}(z)=-2^{1-n_{a}} \sum_{\mathfrak{s} \in \operatorname{Spin}^{c}(X)} \#\left(\bar{V}(z) \cap \bar{W}\left(x^{n_{a}-1}\right) \cap \mathbf{L}_{W, E, L_{1}}\right) .
$$


This is a sum over the finite set of $\operatorname{spin}^{c}$ structures $\mathfrak{s}=\mathfrak{s}_{0} \otimes L_{1}$ with a nonempty SW moduli space $M_{\mathfrak{s}}^{s w}$, defining reducible $\mathrm{PU}(2)$ monopoles contained in $\bar{M}_{W, E}$. The definition of the links $\mathbf{L}_{W, E, L_{1}}$ and the proof that the intersection numbers in Equation (3.5) are well-defined appears in [9]. If we assume the following conjecture, the sum in Equation (3.5) can be reduced to a sum over $\operatorname{spin}^{c}$ structures $\mathfrak{s}$ with $S W_{X}(\mathfrak{s}) \neq 0$.

Conjecture 3.1. Let $X$ be a closed, oriented, smooth four-manifold with $b_{2}^{+}(X)>0$. Let $\mathfrak{s}=\mathfrak{s}_{0} \otimes L_{1}$ be a $\operatorname{spin}^{c}$ structure defined by $\mathfrak{s}_{0}$ and a reduction $E_{-\ell}=L_{1} \oplus(\operatorname{det} E) \otimes L_{1}^{*}$, where $c_{2}\left(E_{-\ell}\right)=c_{2}(E)-\ell$ and $\ell \geq 0$. Let $\mathbf{L}_{W, E, L_{1}}$ be the link of the ideal Seiberg-Witten moduli space $M_{\mathfrak{s}}^{s w} \times \operatorname{Sym}^{\ell}(X)$. Then the intersection number

$$
\#\left(\bar{V}(z) \cap \bar{W}\left(x^{n_{a}-1}\right) \cap \mathbf{L}_{W, E, L_{1}}\right)
$$

appearing in the right-hand side of Equation (3.5) is a multiple of $S W_{X}(\mathfrak{s})$ and thus vanishes if $S W_{X}(\mathfrak{s})=0$.

For reducible $\mathrm{PU}(2)$ monopoles contained in the top level, Conjecture 3.1 follows immediately from Theorem 1.1 in [6] and, when $\ell=1$, from unpublished work of the first and third author [10]. To appreciate why the conjecture should hold more generally, recall that the construction in [8] of the link $\mathbf{L}_{W, E, L_{1}}$ via gluing maps shows that $\mathbf{L}_{W, E, L_{1}}$ is a union of pieces $\mathbf{L}_{\Sigma}$, where $\Sigma$ ranges over the smooth strata of $\operatorname{Sym}^{\ell}(X)$. Each $\mathbf{L}_{\Sigma}$ admits a fiber-bundle structure over $M_{\mathfrak{s}}^{s w} \times \Sigma$. By choosing suitable lifts of the cohomology classes on the link $\mathbf{L}_{W, E, L_{1}}$ to cohomology classes with compact support in the pieces $\mathbf{L}_{\Sigma}$, a pushforward argument shows that each pairing with $\mathbf{L}_{\Sigma}$ can be expressed in terms of a pairing with $M_{\mathfrak{s}}^{s w} \times \Sigma$. The cohomology classes (given by the pushforward) to be paired with $M_{\mathfrak{s}}^{s w} \times \Sigma$ all contain a factor of $h^{d_{s}}$, where $2 d_{s}=\operatorname{dim} M_{\mathfrak{s}}^{s w}$ and $h$ is the element of $H^{2}\left(M_{\mathfrak{s}}^{s w} ; \mathbb{Z}\right)$ used to define the Seiberg-Witten invariant. Thus, all the pairings would be multiples of $S W_{X}(\mathfrak{s})$. This argument is discussed further in [2, pp. 141-142] for the case of a zero-dimensional moduli space $M_{\mathfrak{s}}^{s w}$.

If we did not assume the conjecture, the conditions on $\Lambda$ necessary to ensure that the reducible points in $\bar{M}_{W, E}$ appear only in the top level would be much stricter than those of Theorem 2.1 (and generally unverifiable). Not only would we need to require that $\Lambda \cdot c_{1}(\mathfrak{s})=0$ for all $\mathfrak{s}$ with $M_{\mathfrak{s}}^{s w}$ non-empty (rather than just $\mathfrak{s}$ with $S W_{X}(\mathfrak{s}) \neq 0$ ), but the presence of positive-dimensional, non-empty Seiberg-Witten moduli spaces would further reduce the degree of Donaldson invariant computable by Theorem 2.1 (see the explanation of the condition $\delta \leq$ $r(\Lambda)$ in $\S 3.2$ below). Finally, moduli spaces $M_{\mathfrak{s}}^{s w}$ with $S W_{X}(\mathfrak{s})=0$ may or may not be empty, depending on the choice of perturbations, and - except for some special cases [21] — it appears very difficult to choose perturbations in such a way that those moduli spaces would be empty; moreover, the perturbations of the Seiberg-Witten equations arising as reductions of $\mathrm{PU}(2)$ monopole equations are constrained by the choice of perturbations in the latter equations $[1],[5],[6]$. 
3.2. Comparing indices and degrees. Continue the notation of $\S 3.1$. To apply Theorem 1.1 in [6], given Conjecture 3.1, we need to check that the following conditions are obeyed:

- The $\operatorname{spin}^{c}$ structures $\mathfrak{s}$ with non-zero Seiberg-Witten invariants define reducible $\mathrm{PU}(2)$ monopoles which are contained only in the top level of $\bar{M}_{W, E}$, if at all, and

- The index $n_{a}=\operatorname{Index}_{\mathbb{C}} D_{A}$ is positive, where $D_{A}: \Gamma\left(W^{+} \otimes E\right) \rightarrow$ $\Gamma\left(W^{-} \otimes E\right)$ is the Dirac operator appearing in the $\mathrm{PU}(2)$ monopole equations.

We now show how these conditions are equivalent to the constraints on $\delta, r(\Lambda)$ and $i(\Lambda)$ in the hypotheses of Theorem 2.1.

Proof that Theorem 1.1 in [6] and Conjecture 3.1 implies Theorem 2.1. For $\mathfrak{s} \in S$, let $\mathfrak{s}$ (and the choice of $W^{+}$) define a Hermitian, rank-two vector bundle $E^{\prime}$ with $c_{1}\left(E^{\prime}\right)=c_{1}(E)$, as in $\S 3.1$. We have $c_{1}(\mathfrak{s})^{2}=2 \chi(X)+3 \sigma(X)$ by Equation (1.3) (since $X$ has SW-simple type) and $c_{1}(\mathfrak{s}) \cdot \Lambda=0$ (by choice of $\Lambda$ ), so the formula $(3.2)$ for $p_{1}\left(\mathfrak{s u}\left(E^{\prime}\right)\right)$ gives

$$
p_{1}\left(\mathfrak{s u}\left(E^{\prime}\right)\right)=\left(c_{1}(\mathfrak{s})-\Lambda\right)^{2}=2 \chi(X)+3 \sigma(X)+\Lambda^{2} .
$$

By the definition (3.3) of $\ell$, the identity (3.1) for $\delta$ (with $p=p_{1}(\mathfrak{s u}(E))$ ), and the definition $(2.3)$ of $r(\Lambda)$, we have

$$
\begin{aligned}
4 \ell & =p_{1}\left(\mathfrak{s u}\left(E^{\prime}\right)\right)-p_{1}(\mathfrak{s u}(E)) \\
& =\delta+\Lambda^{2}+\frac{1}{4}(11 \chi(X)+15 \sigma(X)) \\
& =\delta-r(\Lambda) .
\end{aligned}
$$

Therefore, $M_{\mathfrak{s}}^{s w}$ is contained in $\bar{M}_{W, E}$ only if $\delta \geq r(\Lambda)$, in which case it lies in the level

$$
\ell(\delta, \Lambda)=\frac{1}{4}(\delta-r(\Lambda))
$$

Note that in arriving at the formula for $\ell(\delta, \Lambda)$, we only used the facts that $c_{1}(\mathfrak{s})^{2}=2 \chi(X)+3 \sigma(X)$ and $c_{1}(\mathfrak{s}) \cdot \Lambda=0$, for $\mathfrak{s} \in S(X)$ : thus if any one SWbasic class $c_{1}(\mathfrak{s})$ defines a reducible in the level $\ell$ of $\bar{M}_{W, E}$, then all the reducibles associated to SW-basic classes are contained in this level.

The second condition necessary to apply [6, Theorem 1.1] is that the index of the Dirac operator, $D_{A}$, be positive. Using the identity

$$
\operatorname{Index}_{\mathbb{C}} D_{A}=\frac{1}{4}\left(p_{1}(\mathfrak{s u}(E))+\Lambda^{2}-\sigma(X)\right),
$$

and the identity (3.1) for $\delta$, together with the definition (2.4) of the parameter $i(\Lambda)$, one sees that

$$
\operatorname{Index}_{\mathbb{C}} D_{A}=\frac{1}{4}(i(\Lambda)-\delta)
$$


Hence, the condition $\delta<i(\Lambda)$ in the hypotheses of Theorem 2.1 is equivalent to Index $_{\mathbb{C}} D_{A}>0$ and so we can apply Theorem 1.1 in [6].

Since $\delta \leq r(\Lambda)$, the $\operatorname{spin}^{c}$ structures $\mathfrak{s}$ defining basic classes give SeibergWitten moduli spaces $M_{\mathfrak{s}}^{s w}$ and reducible $\mathrm{PU}(2)$ monopoles contained only in the top level of $\bar{M}_{W, E}$. The only other possible contributions to the Donaldson invariants $D_{X}^{w}\left(h^{\delta-2 m} x^{m}\right)$ would be due to pairings of cohomology classes with links of Seiberg-Witten moduli spaces $M_{\mathfrak{s}^{\prime}}^{s w}$ defining reducible $\mathrm{PU}(2)$ monopoles contained in levels $\ell \geq 1$ of $\bar{M}_{W, E}$. As such classes $c_{1}\left(\mathfrak{s}^{\prime}\right)$ are not basic, we have $S W_{X}\left(\mathfrak{s}^{\prime}\right)=0$ and so Conjecture 3.1 implies that the moduli spaces $M_{\mathfrak{s}^{\prime}}^{s w}$ make no contribution to the Donaldson invariant $D_{X}^{w}\left(h^{\delta-2 m} x^{m}\right)$.

In Equation (2.5) of Theorem 2.1, which asserts the vanishing of Donaldson invariants through certain degrees, one only needs the more elementary Theorem 3.33 from [6] - as it is not necessary to evaluate the pairings on the right-handside - rather than the more difficult Theorem 1.1 from [6] which is needed to compute the formula (2.6) for Donaldson invariants.

Without the assumption that $X$ have SW-simple type, we could at best write

$$
c_{1}(\mathfrak{s})^{2} \leq 2 \chi(X)+3 \sigma(X)+4 d(X)
$$

where $d(X)$ is the maximal expected dimension of the Seiberg-Witten moduli spaces corresponding to $\mathfrak{s} \in S$. The results of $\S 1$ could then be replaced with weaker ones, where the order of vanishing would now also depend on $d(X)$.

In the absence of Conjecture 3.1, we would need to replace the set $S$ in the statement of Theorem 2.1 by the subset of $H^{2}(X ; \mathbb{Z})$ corresponding to $\operatorname{spin}^{c}$ structures with non-empty Seiberg-Witten moduli spaces. Furthermore, the SW-simple type equation for $c_{1}(\mathfrak{s})^{2}$ would have to be replaced by the weaker inequality described in the preceding remark, but with $d(X)$ now denoting the maximal dimension of the non-empty Seiberg-Witten moduli spaces. (In this case, $d(X)$ is no longer independent of the choice of generic perturbation parameters.)

\section{References}

[1] P. M. N. Feehan, Generic metrics, irreducible rank-one PU(2) monopoles, and transversality, to appear in Comm. Anal. Geom., math.DG/9809001.

[2] P. M. N. Feehan and T. G. Leness, PU(2) monopoles and relations between four-manifold invariants, Topology Appl. 88 (1998), 111-145, dg-ga/9709022.

[3] — PU(2) monopoles. I: Regularity, Uhlenbeck compactness, and transversality, J. Differential Geom. 49 (1998), 265-410, dg-ga/9710032.

[4] _ PU(2) monopoles. II: Highest-level singularities and relations between fourmanifold invariants, first version of [5] and [6], December 8, 1997; dg-ga/9712005 (v1).

[5] $\longrightarrow$ PU(2) monopoles and links of top-level Seiberg-Witten moduli spaces, preprint $(\S 1-3$ of $[4])$.

[6] _ PU(2) monopoles. II: Top-level Seiberg-Witten moduli spaces and a special case of Witten's conjecture, preprint (§4-7 of [4]).

[7] _ Donaldson invariants and wall-crossing formulas. I: Continuity of gluing and obstruction maps, submitted to a print journal, math.DG/9812060. 
[8] _ , PU(2) monopoles. III: Existence and continuity of gluing and obstruction maps, in preparation.

[9] _ PU(2) monopoles. IV: Surjectivity of gluing maps, in preparation.

[10] , in preparation.

[11] R. Fintushel and R. Stern, Donaldson invariants of 4-manifolds with simple type, J. Differential Geom. 42 (1995), 577-633.

[12] _ Immersed spheres in 4-manifolds and the immersed Thom conjecture, Turkish J. Math. 19 (1995), 145-157.

[13] Nonsymplectic 4-manifolds with one basic class, preprint.

[14] R.E. Gompf and T.S.Mrowka, Irreducible four-manifolds need not be complex, Ann. of Math. (2) 138 (1993), 61-111.

[15] D. Kotschick, $S O(3)$ invariants for four-manifolds with $b^{+}=1$, Proc. London Math. Soc. 63 (1991), 426-448.

[16] P. B. Kronheimer and T. S. Mrowka, Embedded surfaces and the structure of Donaldson's polynomial invariants, J. Differential Geom. 43 (1995), 573-734.

[17] M. Mariño, G. Moore, and G. Peradze, Four-manifold geography and superconformal symmetry, math.DG/9812042.

[18] _ Superconformal invariance and the geography of four-manifolds, hep-th/9812055.

[19] J. W. Morgan and T. S. Mrowka, A note on Donaldson's polynomial invariants, Internat. Math. Res. Notes 10 (1992), 223-230.

[20] V. Y. Pidstrigach and A. N. Tyurin, Localisation of Donaldson invariants along the Seiberg-Witten classes, dg-ga/9507004.

[21] E. Witten, Monopoles and four-manifolds, Math. Res. Lett. 1 (1994), 769-796, hep-th/9411102.

Department of Mathematics, Ohio State University, Columbus, OH 43210

Current address: School of Mathematics, Institute for Advanced Study, Olden Lane, Princeton, NJ 08540

E-mail address: feehan@math.ohio-state.edu and feehan@math.ias.edu

Department of Mathematics, Harvard University, 1 Oxford Street, Cambridge, MA 02138

E-mail address: kronheim@math.harvard.edu

Department of Mathematics, Florida International University, Miami, FL 33199 E-mail address: lenesst@fiu.edu

Department of Mathematics, rm 2-367, Massachusetts Institute of Technology, CAMBridge, MA 02139

E-mail address: mrowka@math.mit.edu 